\title{
Stand der Forschung
}

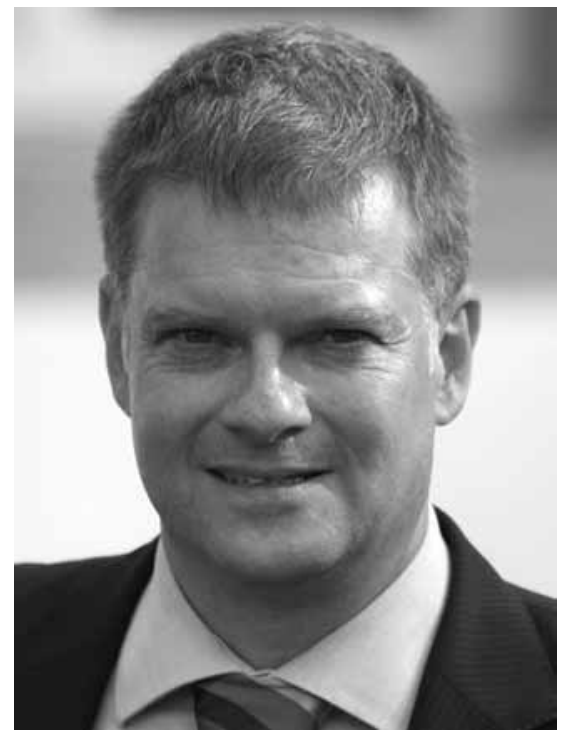

Sowohl in der Informationssicherheit als auch im Datenschutz ist der "Stand der Technik" zunehmend das Maß, mit dem die Eignung und die Angemessenheit von Schutzmaßnahmen bewertet werden: Unter diesem Niveau sollte man nicht bleiben, will man sich nicht den Vorwurf der Fahrlässigkeit gefallen lassen, und darüber hinaus sollte man nicht gehen, um keine unnötigen Aufwände vorgehalten zu bekommen. Die Bestimmung dieser Eignungsgrenze ist in der Praxis allerdings durchaus nicht einfach. In Heft 11/2017 hat sich Michael Knopp dieser Frage aus juristischer Perspektive genähert, und in dieser Ausgabe ergänzen Detlef Weidenhammer und Rocco Gundlach seine Überlegungen aus einem technischen Blickwinkel.

Da der "Stand der Technik" aber auch ständiger Weiterentwicklung unterliegt, ist es von Zeit zu Zeit sinnvoll, einen vorsichtigen Blick in die Zukunft zu werfen: Welche Entwicklungen sind aus der Forschung zu erwarten? Denn bei hinreichender Praxistauglichkeit darf damit gerechnet werden, dass Forschungsergebnisse in absehbarer Zeit Einzug in Produkte und Lösungen halten - und damit für eine Anpassung der Definition des "Stands der Technik" sorgen. Für dieses Heft haben wir daher nachgefragt, welchen Fragen die Sicherheits- und Datenschutzforschung in Deutschland nachgeht.

Das Bild, das die sechs Beiträge dieses Schwerpunkthefts zeichnen, ist natürlich unvollständig - daher werden wir die Frage im Laufe des Jahres in einem weiteren Schwerpunkt erneut aufgreifen. Allerdings geben die Beiträge eine Vorstellung von der Vielfalt und den interessanten Richtungen, die in der Sicherheits- und Datenschutzforschung derzeit verfolgt werden.

Einer dieser Ansätze ist die Nutzung von Digitalen Wasserzeichen in der Forensik, der von Michael Waschetzki und Martin Steinebach verfolgt wird. Das Forschungsteam um Jörn Müller-Quade (Brandon Broadnax, Bernhard Löwe, Jeremias Mechler und Matthias Nagel) arbeitet an der Verankerung von Sicherheitsmechanismen in Hardware, einem zentralen Fundament, das gerne aus dem Blick gerät. Stefan Nürnberger blickt hinter die Kulissen der Verarbeitung von Fahrzeugdaten - absehbar eine Herausforderung für Datenschützer. Ninja Marnau, Pascal Berrang und Mathias Humbert untersuchen die Anonymisierbarkeit von genetischen Daten: Eine Aufgabenstellung mit erheblichem Risikopotential für die Betroffenen. Datenschutzrechtlich ebenfalls herausfordernd: Die Frage der Zulässigkeit sozialwissenschaftlicher Forschung in Sozialen Netzwerken, die Sebastian Golla, Henning Hofmann und Matthias Bäcker untersuchen. Und abschließend geben Bernhard Esslinger und Hernâni Marques einen Einblick in das Konzept hinter pretty Easy privacy ( $p E p$ ) - den Versuch, die Nutzung von Verschlüsselung ohne Verlust an Sicherheit einfach und bequem zu gestalten.

\section{Dirk Fox}

